\title{
Melt-processable Nacre-mimetic Hydrocarbon
}

\author{
Composites via Polymer 1D Nanostructure
}

\section{Formation}

Fan Zhong', Ralf Thomann' ${ }^{1}$, Yi Thomann ${ }^{1}$, Laura Burk' ${ }^{1}$, Rolf Mülhaupt ${ }^{\text {* }}$

${ }^{1}$ Freiburg Materials Research Center (FMF) and Institute for Macromolecular

Chemistry of the Albert-Ludwigs-University Freiburg, Stefan-Meier-Strasse 31, D-

79104, Germany

Fax: (+49) 761203 6319; 


\section{Supporting Information}

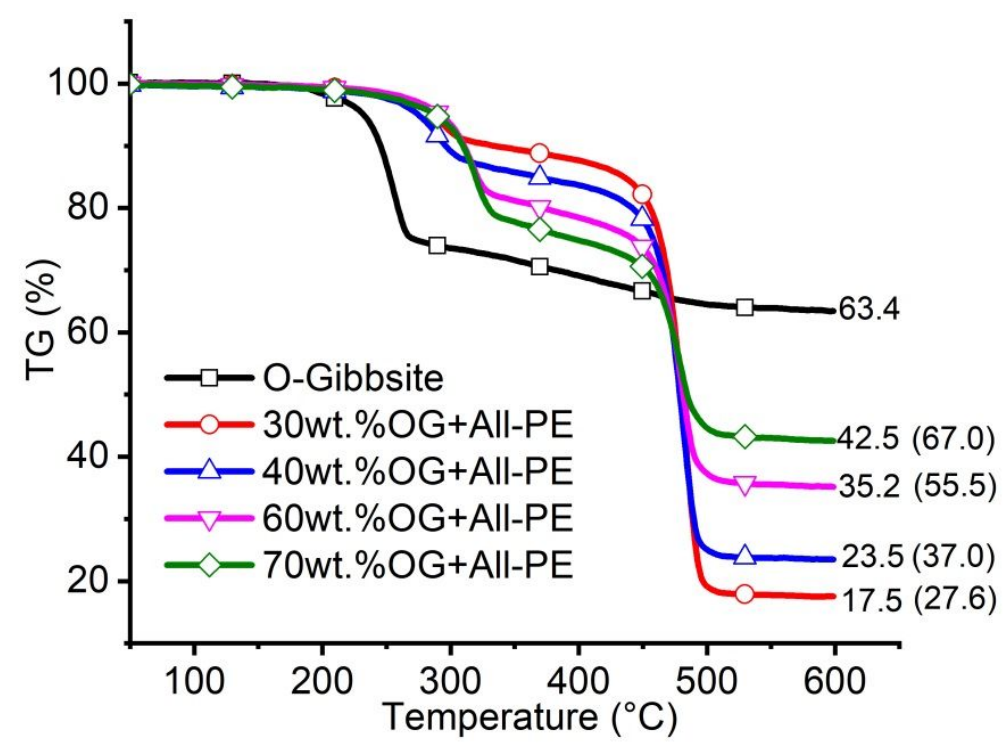

S-1. The real weight fraction of incorporated O-gibbsite, calculated by comparison with

O-gibbsite, confirmed by TGA in the temperature range from $50{ }^{\circ} \mathrm{C}$ to $600{ }^{\circ} \mathrm{C}$. In addition, a massive mass loss by the splitting off of water occurred at approximately $300{ }^{\circ} \mathrm{C}$. 


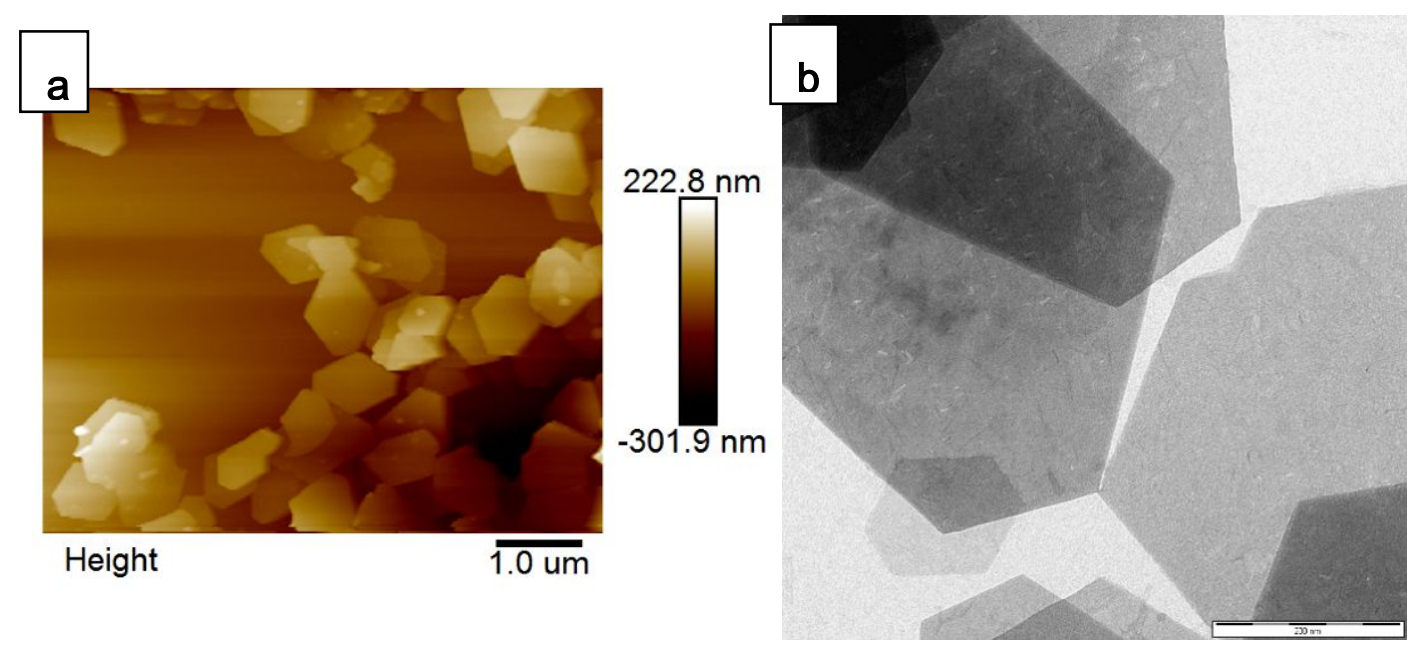

S-2. a) AFM height imaging of nonmodified gibbsite. b) TEM image of nonmodified gibbsite with a scale bar of $200 \mathrm{~nm}$.
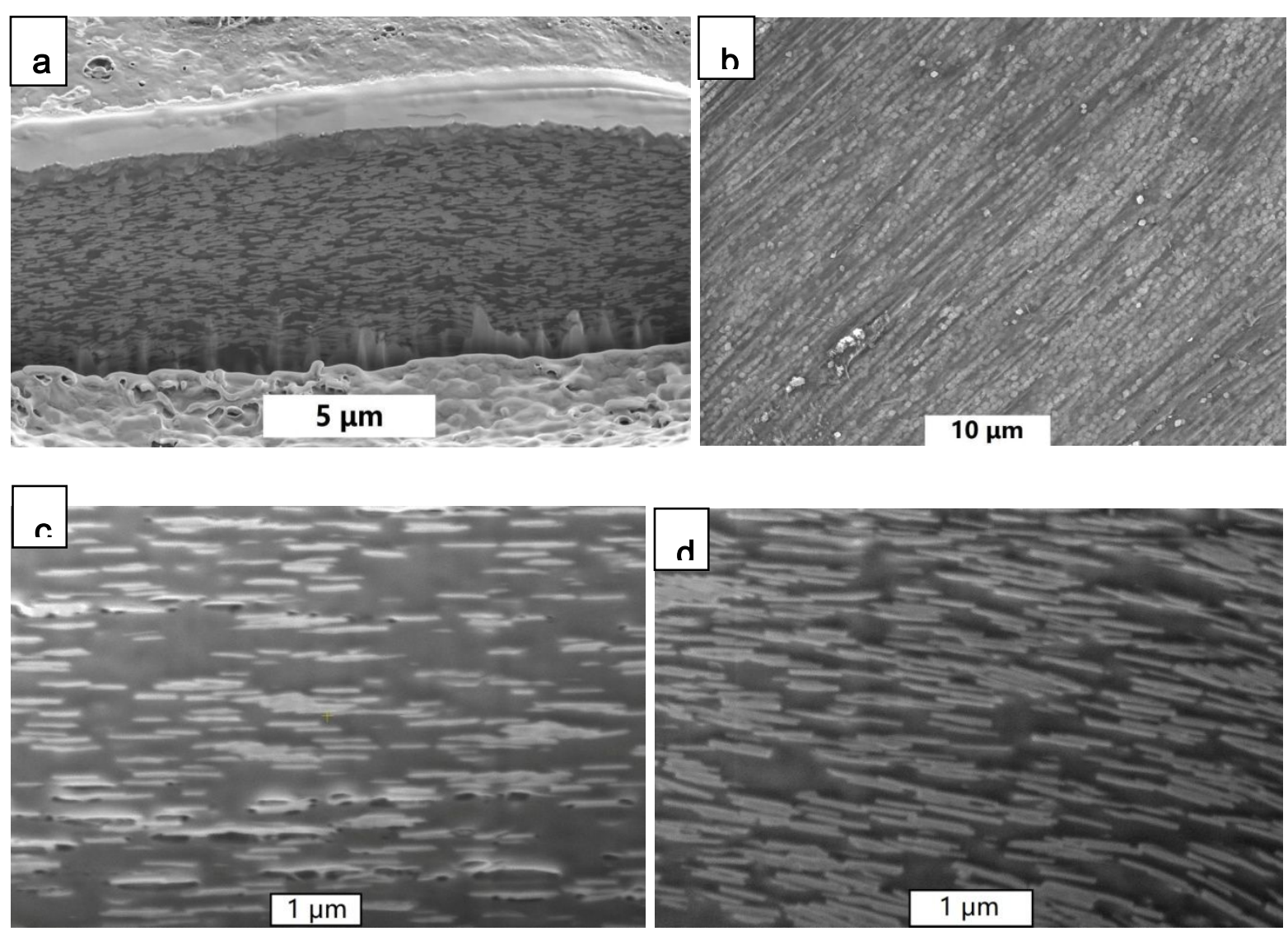
S-3. a) FIB-SEM side-view image of the whole cross-section of 70 wt. \% OG+All-PE;

b) ESEM top view of 70 wt.\% OG+All-PE, showing a uniform distribution of O-gibbsite;

c) FIB-SEM side-view image of 40 OG+All-PE; d) FIB-SEM side-view image of 60

OG+All-PE.
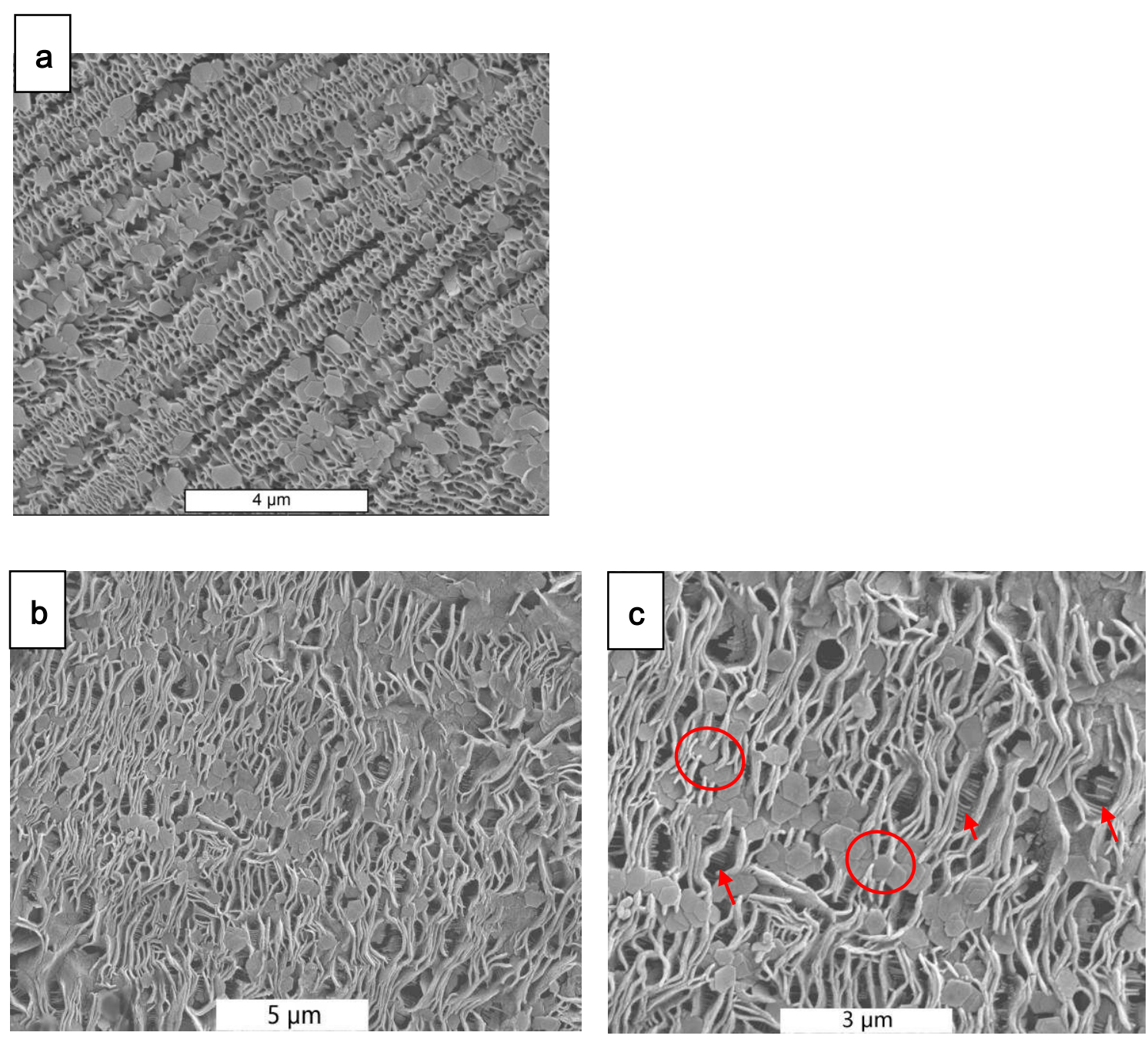

S-4. The shish-kebab structure of 70 wt. $\%$ OG+All-PE and 30 wt. \% OG+All-PE was confirmed by SEM at different magnifications: a) 70 wt. $\%$ OG+All-PE $(4 \mu \mathrm{m})$ b) $30 \mathrm{wt} . \%$ 
OG+All-PE $(5 \mu \mathrm{m}) \mathrm{c}) 30 \mathrm{wt} . \%$ OG+All-PE $(3 \mu \mathrm{m})$

The diameter of kebabs (30 wt.\% OG+All-PE) is approximately $2 \mu \mathrm{m}$, which is larger than those in $70 \mathrm{wt.} \%$ OG+All-PE $(500 \mathrm{~nm})$. In addition, multi-shish features and intercalated O-gibbsite in kebabs are marked with red arrows and circles, respectively, in the right image.

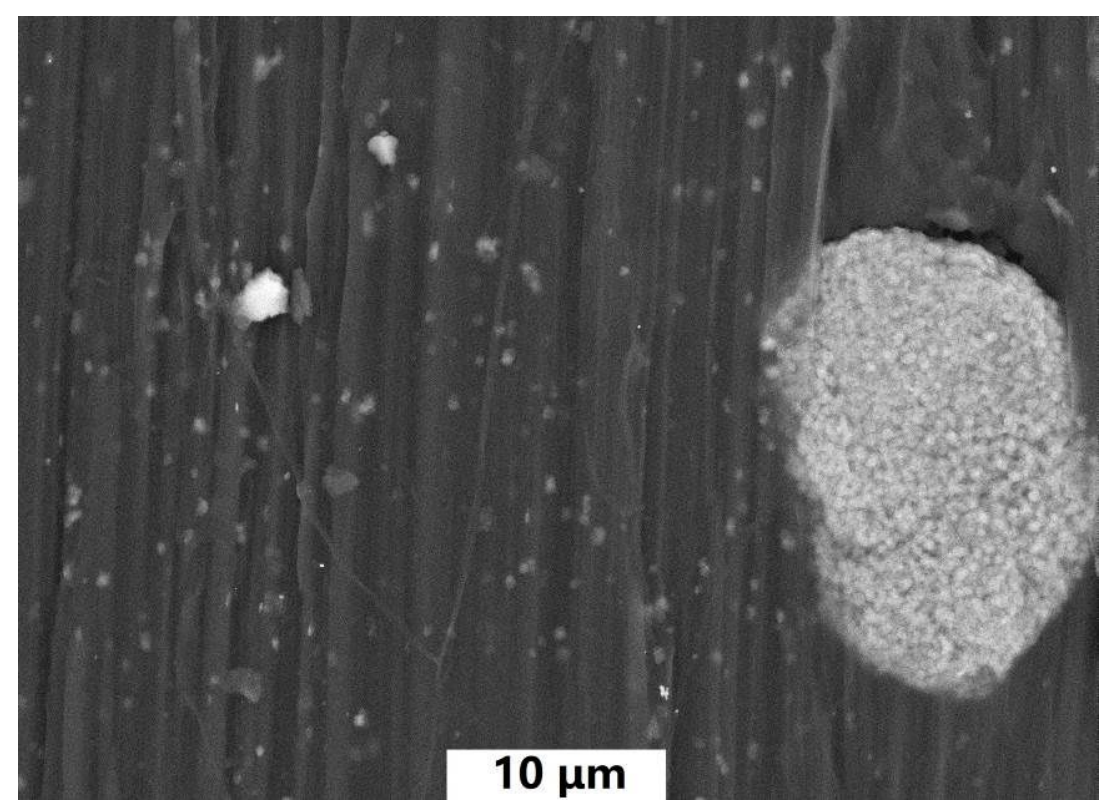

S-5: SEM image of the sample with 5 wt.\% nonmodified gibbsite. Gibbsite formed aggregates during processing, which is not suitable for replicating the nacre structure. 


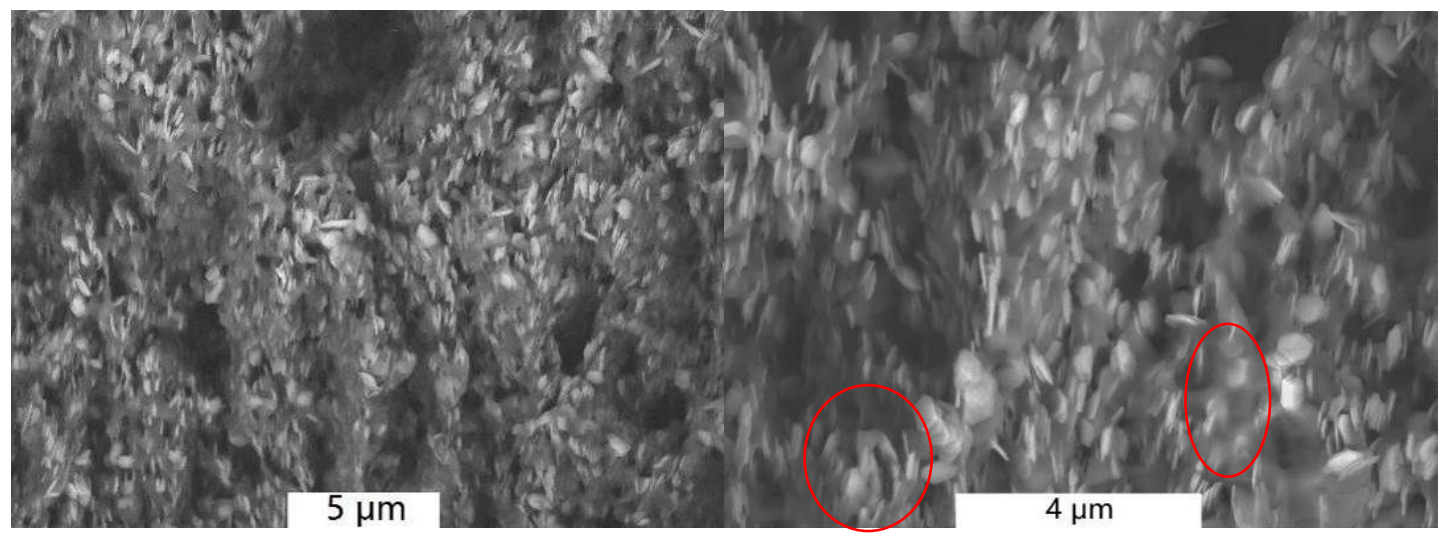

S-6: SEM images at two different magnifications of the fracture surface after the tensile test. A rough fracture surface is observed due to the plastic deformation of $\mathrm{PE}$, and Ogibbsite was partially removed from the polymer without being broken.

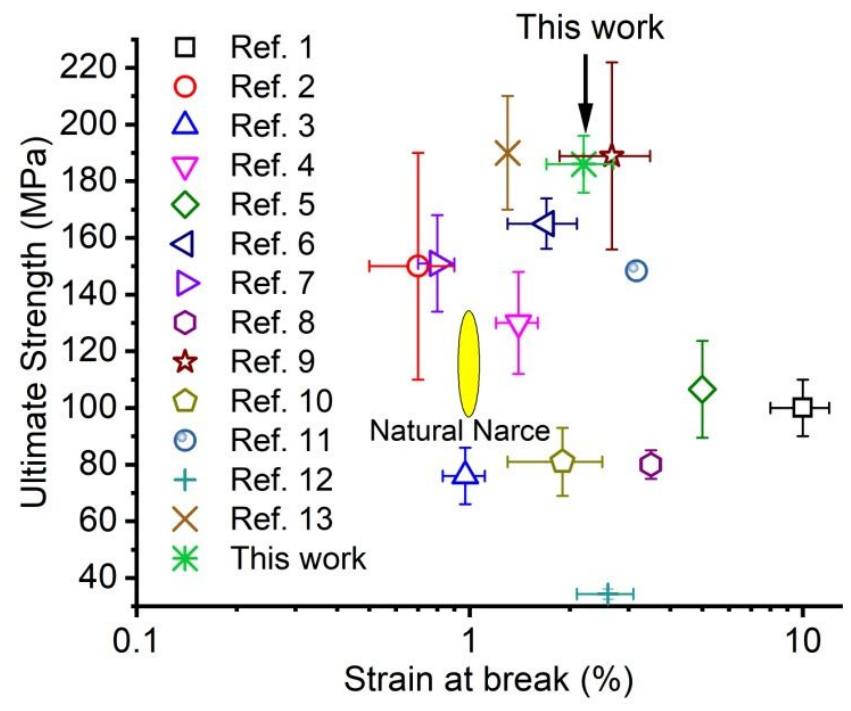

S-7: Comparing the mechanical properties of 70 wt. $\%$ OG+All-PE with some presented results. 
1. Tang, Z., Kotov, N. A., Magonov, S. \& Ozturk, B. Nanostructured artificial nacre. Nature materials 2, 413-418; 10.1038/nmat906 (2003).

2. Podsiadlo, P. et al. Ultrastrong and stiff layered polymer nanocomposites. Science (New York, N.Y.) 318, 80-83; 10.1126/science.1143176 (2007).

3. Yao, H.-B., Tan, Z.-H., Fang, H.-Y. \& Yu, S.-H. Artificial nacre-like bionanocomposite films from the self-assembly of chitosan-montmorillonite hybrid building blocks. Angewandte Chemie (International ed. in English) 49, 10127-10131; 10.1002/anie.201004748 (2010)

4. An, Z., Compton, O. C., Putz, K. W., Brinson, L. C. \& Nguyen, S. T. Bio-inspired borate cross-linking in ultra-stiff graphene oxide thin films. Advanced materials (Deerfield Beach, Fla.) 23, 3842-3846; 10.1002/adma.201101544 (2011).

5. Cheng, Q., Wu, M., Li, M., Jiang, L. \& Tang, Z. Ultratough artificial nacre based on conjugated cross-linked graphene oxide. Angewandte Chemie (International ed. in English) 52, 3750-3755; 10.1002/anie.201210166 (2013).

6. Walther, A. et al. Large-area, lightweight and thick biomimetic composites with superior material properties via fast, economic, and green pathways. Nano letters 10, 2742-2748; 10.1021/nl1003224 (2010).

7. Walther, A. et al. Supramolecular control of stiffness and strength in lightweight high-performance nacre-mimetic paper with fire-shielding properties. Angewandte Chemie (International ed. in English)49, 6448-6453; 10.1002/anie.201001577 (2010). 8. Chen, R., Wang, C.-a., Huang, Y. \& Le, H. An efficient biomimetic process for fabrication of artificial nacre with ordered-nanostructure. Materials Science and 
Engineering: C 28, 218-222; 10.1016/j.msec.2006.12.008 (2008).

9. Li, Y.-Q., Yu, T., Yang, T.-Y., Zheng, L.-X. \& Liao, K. Bio-inspired nacre-like composite films based on graphene with superior mechanical, electrical, and biocompatible properties. Advanced materials (Deerfield Beach, Fla.) 24, 3426-3431; 10.1002/adma.201200452 (2012).

10. Podsiadlo, P., Tang, Z., Shim, B. S. \& Kotov, N. A. Counterintuitive effect of molecular strength and role of molecular rigidity on mechanical properties of layer-bylayer assembled nanocomposites. Nano letters 7, 1224-1231; 10.1021/nl0700649 (2007).

11. Putz, K. W., Compton, O. C., Palmeri, M. J., Nguyen, S. T. \& Brinson, L. C. High-Nanofiller-Content Graphene Oxide-Polymer Nanocomposites via VacuumAssisted Self-Assembly. Adv. Funct. Mater. 20, 3322-3329; 10.1002/adfm.201000723 (2010).

12. Bonderer, L. J., Feldman, K. \& Gauckler, L. J. Platelet-reinforced polymer matrix composites by combined gel-casting and hot-pressing. Part I. Polypropylene matrix composites. Composites Science and Technology 70, 1958-1965; 10.1016/j.compscitech.2010.07.014 (2010).

13. Morits, M. et al. Toughness and Fracture Properties in Nacre-Mimetic Clay/Polymer Nanocomposites. Adv. Funct. Mater. 27, 1605378; 10.1002/adfm.201605378 (2017). 


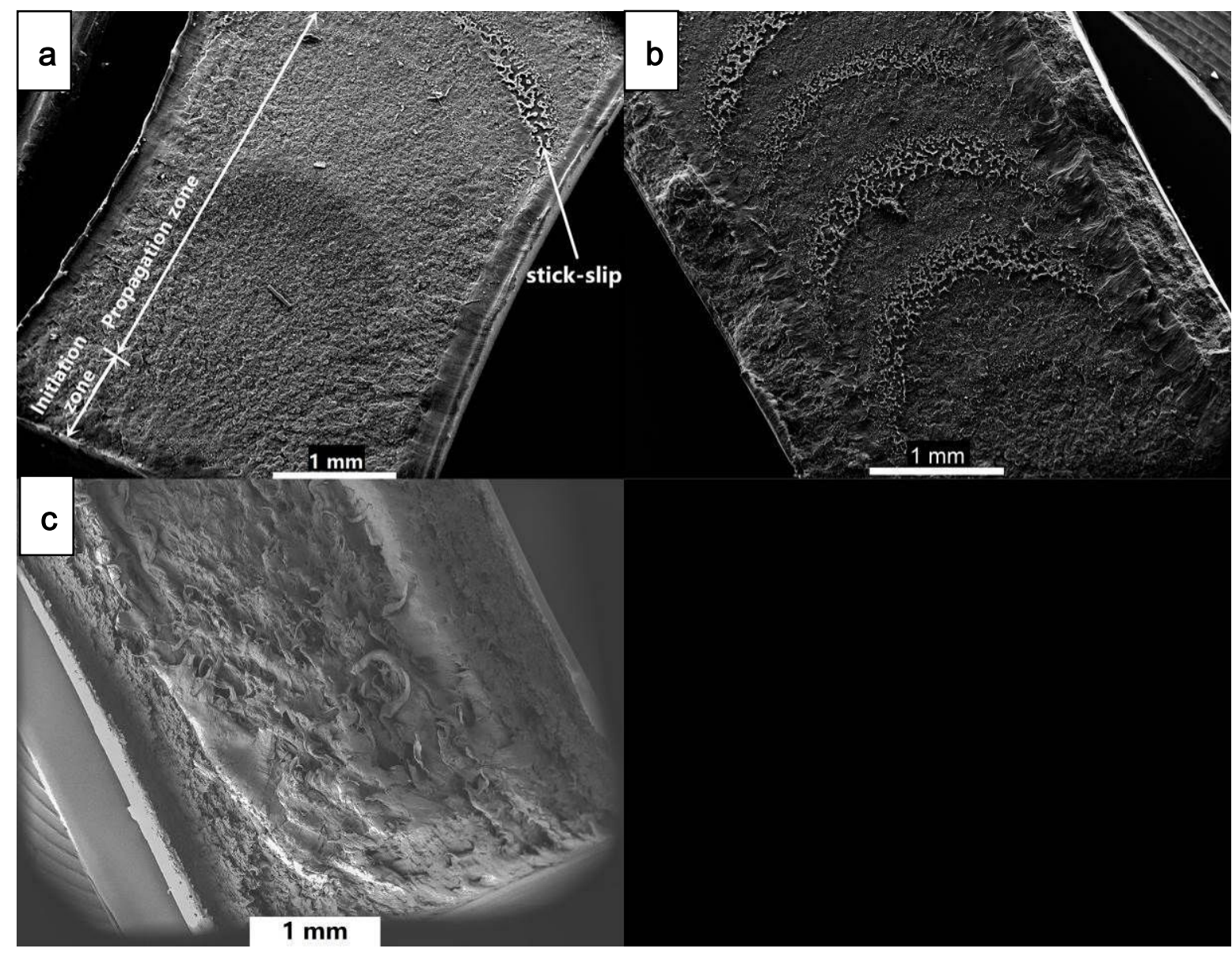

S-8. The fracture surface after notched Izod impact strength was characterized by SEM. a)The smooth fracture surface of HDPE includes an initiation zone, propagation zone and stick-slip zone; b) The All-PE composite exhibits broad and repeated stickslip zones; c) 70 wt.\% OG+All-PE shows a much rougher fracture surface than do the other composites owing to millimeter-sized plastic deformation. 